\title{
A System-Performance-Based Comparison of Sparse Regular and Irregular Antenna Arrays for Millimeter-Wave Multi-User MIMO Base Stations
}

\author{
Roel X. F. Budé ${ }^{1, *(\mathbb{D})}$, Thomas A. H. Bressner $\left.{ }^{2} \mathbb{(}\right)$, Martin N. Johansson ${ }^{3}\left(\mathbb{D}\right.$, Marianna V. Ivashina ${ }^{4} \mathbb{C}^{(}$, \\ Adrianus Bernardus Smolders ${ }^{1}\left(\mathbb{D}\right.$ and Ulf Johannsen ${ }^{1}$ (D) \\ 1 Electrical Engineering Department, Eindhoven University of Technology, \\ 5612 AZ Eindhoven, The Netherlands; a.b.smolders@tue.nl (A.B.S.); u.johannsen@tue.nl (U.J.) \\ 2 Ericsson R\&D Electronics, Ericsson Antenna Technology Germany GmbH, 83026 Rosenheim, Germany; \\ thomas.bressner@ericsson.com \\ 3 Ericsson Research, Ericsson AB, 41756 Gothenburg, Sweden; martin.n.johansson@ericsson.com \\ 4 Electrical Engineering Department, Chalmers University of Technology, 41296 Gothenburg, Sweden; \\ marianna.ivashina@chalmers.se \\ * Correspondence: r.x.f.bude@tue.nl
}

\section{check for}

updates

Citation: Budé, R.X.F.; Bressner,

T.A.H.; Johansson, M.N.; Ivashina,

M.V.; Smolders, A.B; Johannsen, U. A

System-Performance-Based

Comparison of Sparse Regular and

Irregular Antenna Arrays for

Millimeter-Wave Multi-User MIMO

Base Stations. Electronics 2022, 11, 335

https://doi.org/10.3390/

electronics11030335

Academic Editor: Emilio Arnieri

Received: 20 December 2021

Accepted: 17 January 2022

Published: 21 January 2022

Publisher's Note: MDPI stays neutral with regard to jurisdictional claims in published maps and institutional affiliations.

Copyright: () 2022 by the authors. Licensee MDPI, Basel, Switzerland. This article is an open access article distributed under the terms and conditions of the Creative Commons Attribution (CC BY) license (https:/ / creativecommons.org/licenses/by/ $4.0 /)$.

\begin{abstract}
A system-level study was conducted that evaluated the system performance of various dense and sparse antenna array configurations for application in millimeter-wave multi-user multipleinput multiple-output base stations. The performance was evaluated by investigating the probability that a user experiences an outage when a zero-forcing pre-coder is used in a random line of sight scenario. This paper shows that the outage probability significantly decreased when irregular sparse arrays were used rather than regular sparse or regular dense arrays. A re-configurable linear array was designed and realized as a demonstrator. It used 3D-printed aluminum box horn antenna elements that had wide scanning range in the azimuthal plane and a small scanning range in the elevation plane. For the demonstrator, it was shown that the outage probability was reduced from $3.85 \%$ to $0.64 \%$ by moving from a sparse regularly spaced array to a sparse randomly spaced array. This amounted to an improvement of a factor of six. The sparse topology allowed for the usage of large antenna elements that had an increased gain and still achieved wide-angle scanning, while reducing mutual coupling to a minimum.
\end{abstract}

Keywords: 5G; mmWave; antenna arrays; zero-forcing; sparse arrays; MU-MIMO; base stations

\section{Introduction}

The amount of data transferred over wireless networks and the number of users connected to wireless networks are rapidly increasing. The total network traffic has consistently increased by $50 \%$ or more year on year, exceeding 66 EB per month in Q1 of 2021 [1]. This rapid increase is not expected to slow down any time soon. To allow this trend to continue, it is necessary to increase the throughput of mobile networks. This is one of the things that Fifth-Generation Wireless (5G) aims to bring. 5G operates on several frequency bands, but the highest data rates in the order of gigabits per second (Gbit/s) are achieved at millimeter-Wave (mmWave) frequencies, referring to wavelengths in the order of millimeters (20-300 GHz). At these frequencies, a large amount of spectrum is available, which allows for the usage of large bandwidths.

At mmWave, the high path loss and attenuation by objects such as buildings or trees necessitate the deployment of antenna systems with a high effective isotropic radiated power (EIRP). For this purpose, beamforming antenna arrays are proposed at the base stations. Beamforming arrays consist of many antenna elements, which are excited in such a way that a beam is created that is pointed toward the user. 
Other than increasing the EIRP, beamformers have another benefit. If they are equipped with more than one digital channel, it becomes possible to create several simultaneous spatial beams. Each beam can be modulated with a different data stream. As such, a beam can be generated per user and modulated by the data of only that user, allowing several users to communicate simultaneously. At the same time, each user can receive several simultaneous data streams, further increasing the data rate. This concept is called multiple-user multiple-input multiple-output (MU-MIMO).

The excitation weights for each antenna element in the array to achieve MU-MIMO operation are found using a pre-coder. A well-known pre-coder is zero-forcing (ZF). The ZF pre-coder is used to find the excitation weights that force the interference between simultaneous users to zero, while maximizing the signal power of the wanted signals towards each user [2]. ZF is especially suited to a high-signal-to-noise ratio (SNR) environment, since the effect of noise is neglected with this pre-coder [3].

An important limitation of the concept of using MU-MIMO is that the number of simultaneous users is limited by the number of digital channels. Scaling up the number of digital channels of a base station array antenna is not trivial as the implementation complexity increases with the carrier frequency, and the coherence time is decreased at higher carrier frequencies [4]. However, such systems have been demonstrated in practice already. For example, in [5], a 64-element digital beamforming array was demonstrated, achieving a spectral efficiency of up to $101.5 \mathrm{bit} / \mathrm{s} / \mathrm{Hz}$ on a $500 \mathrm{MHz}$ channel at a carrier frequency of $28 \mathrm{GHz}$. Clearly, we are moving in the direction of large-scale fully digital mmWave beamforming arrays. However, the cost and power usage for such systems are important concerns, and it can be beneficial if the number of channels is reduced. In this paper, we explore arrays with a small number of digital channels. Each antenna element in the array has its own digital channel. We considered eight-element linear arrays, and some observations on a sixteen-element linear array were included.

For the application of ZF to achieve MU-MIMO, multiple investigations into the optimum positions of each antenna element have been conducted. In [6], it was shown that moving from a dense array (inter-element spacing in the order of $0.5 \lambda_{0}$ to $0.7 \lambda_{0}$ ) towards a sparse array (inter-element spacing $>0.7 \lambda_{0}$ ) led to a decrease in the ratio between the highest and lowest radiated per-element power in the array, when the angular separation between two users was small. In [7], it was shown that antennas can be placed in a way that decreased the power variation across the array by placing the elements irregularly, corresponding to the spatial power distribution of the array. Both of these studies showed that a sparse irregular array is beneficial in terms of the power variation between antenna elements.

Next to this, it is worth considering that increasing the minimum spacing between the antenna elements allows for the use of larger antennas, and as such allows for the use of antennas with a higher gain that still achieve wide-angle scanning. Increasing the minimum spacing between antenna elements also decreases the mutual coupling. The mutual coupling has a substantial impact on channel capacity as the number of elements increases for a fixed aperture [8]. In [9], it was demonstrated that employing directive antenna elements with reduced radiation beyond the field of view in multi-panel sparse base station antennas has an advantage in terms of the enhanced sum-rate.

Sparse arrays are a solution to the stringent thermal requirements for air-cooled beamforming systems [10]. Better cooling performance can be acquired for a sparse array than for a dense array [11] because the same amount of thermal power is generated on a larger area. Because more area is available per antenna, it may also become possible to use multiple transistors and combiners such as the one proposed in [12] to increase the radiated power in an efficient manner.

From these perspectives, it becomes clear that a sparse array is an important point of interest, especially when considering a MU-MIMO scenario. A well-known issue with sparse arrays is that they exhibit grating lobes within the field of view (FoV) of the antenna array. For traditional radar systems and phased arrays, grating lobes are avoided by 
keeping the inter-element spacing small. Another way to decrease the level of the grating lobes is by placing the elements irregularly. Good examples are the sunflower array [13] and maximally sparse arrays synthesized through compressive sensing [14].

Decreasing the level of the grating lobes significantly for a large FoV does require a large number of antenna elements. However, as mentioned before, we explored array antennas with a small number of antenna elements in this paper. This means the sparse arrays with only 8-16 elements that we investigated will either have grating lobes within the FoV or a high side lobe level (SLL). In recent works, it was already shown that grating lobes do not have a negative impact on the performance in a MU-MIMO scenario. In [15], it was shown that data streams became less correlated when the inter-element spacing was increased, regardless of the appearance of grating lobes. In [16], a similar finding was obtained, where it was shown that the overall data rate was improved when employing a sparse regular array rather than a dense one. This conclusion was also reached in [17], where in addition to regularly spaced arrays, an irregularly spaced array with 200 antenna elements was considered. Here, it was concluded that irregularly spaced arrays with an aperture as large as possible should be used for massive MIMO antenna array designs.

In this paper, we show that moving from a dense to a sparse irregular antenna array is beneficial in terms of the performance of the full system, even when a small number of antenna elements is used and the irregularity is generated randomly. To this end, we investigated the reliability of the system in terms of the probability that a user experiences a data rate below a certain threshold (an "outage"). Different array topologies were explored and compared; dense arrays, sparse regular, and sparse irregular (including random) antenna arrays.

The novel contributions of this paper are (1) a system-level comparison in terms of the outage probability of regular dense, regular sparse, irregular sparse, and irregular random antenna arrays, (2) the use of medium-gain wide-angle scanning horn antennas in a sparse array antenna, and (3) an experimental verification of this concept using 3Dprinted wide-angle scanning horn antennas in a sparse random configuration with eight antenna elements.

The remainder of the paper is structured as follows. In Section 2, the system model used is given, the scenario chosen, and the measure of performance defined. In Section 3, regular sparse and irregular sparse arrays are compared. In Section 4, several array configurations are investigated in an attempt to determine the optimal array type. In Section 5, a prototype is designed and built using 3D-printed horn antennas with the goal to investigate the difference between regular and irregular antenna arrays in a line of sight (LOS) environment. The discussion and recommendations for future work are given in Section 6. The conclusion is drawn in Section 7.

\section{Model}

As a measure of the performance of the communication system, the probability of a user experiencing an outage was used. The outage is defined here as the case that the user is experiencing a data rate that is below a certain threshold. In [18], it was suggested that the goal be to provide the users with at least $100 \mathrm{Mbit} / \mathrm{s}$. For typical equipment and channel parameters, it can be shown that the minimum carrier-to-interference-plus-noise ratio (CINR) required to achieve a data rate of $100 \mathrm{Mbit} / \mathrm{s}$ is approximately $3 \mathrm{~dB}$ when considering a bandwidth $B$ of $500 \mathrm{MHz}$, QPSK modulation, and no forward error correction. As such, the CINR $=3 \mathrm{~dB}$ threshold was considered in this paper. In extension, this means an outage occurs when the CINR is below $3 \mathrm{~dB}$ for a given array configuration.

\subsection{Channel Model}

In this paper, only the down-link of a $120^{\circ}$ sector of a cell was considered. The base station consisted of a linear array with $N$ antenna elements. In the sector, $K$ users were randomly and uniformly distributed within a minimum distance $r_{\min }$ and a maximum 
distance $r_{\max }$. Only (LoS) conditions were considered. This made it a random LoS scenario. The coordinate system and a schematic overview of the situation are shown in Figure 1.

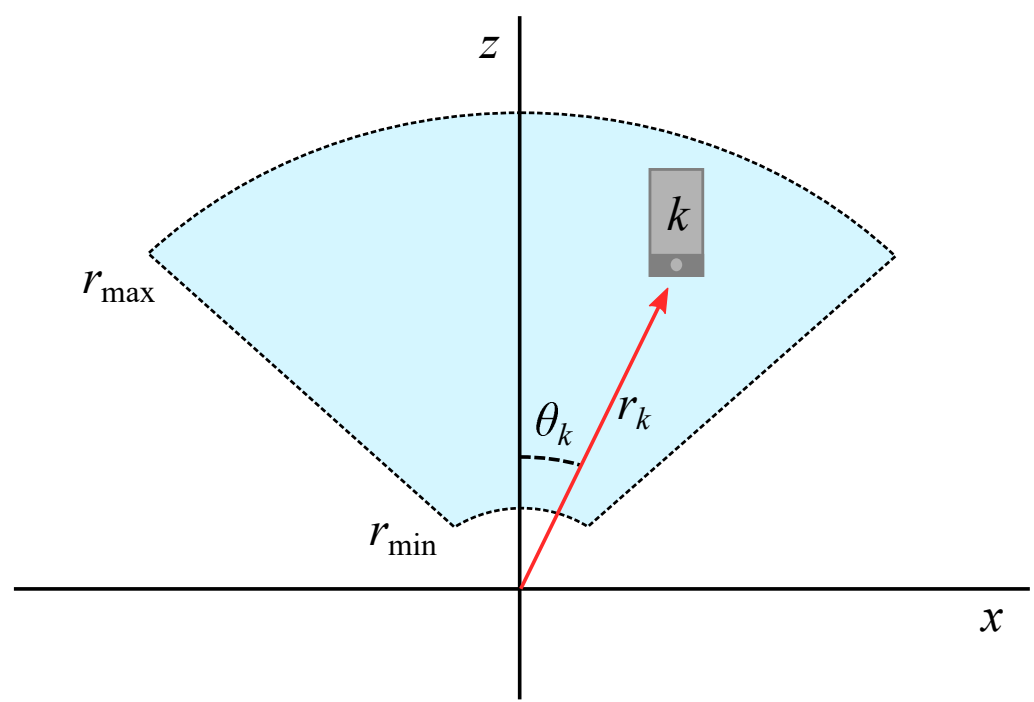

Figure 1. The users are randomly positioned inside the sector ranging from $r_{\min }=10 \mathrm{~m}$ to $r_{\max }=100 \mathrm{~m}$ and within $-60^{\circ} \leq \theta \leq 60^{\circ}$. The $k$ th user is positioned at distance $r_{k}$ and angle $\theta_{k}$. The $N$-element linear array is aligned with the $x$-axis and is located at $x=0$.

The received signal vector $\mathbf{y} \in \mathbb{C}^{K \times 1}$ is given by:

$$
\mathbf{y}=\alpha \mathbf{H W s}+\mathbf{u}
$$

where $\mathbf{s} \in \mathbb{C}^{K \times 1}$ is the transmit vector, $\mathbf{u} \in \mathbb{C}^{K \times 1}$ is the received noise vector, $\mathbf{H} \in \mathbb{C}^{K \times N}$ is the channel matrix, and $\mathbf{W} \in \mathbb{C}^{N \times K}$ is the pre-coding matrix. The parameter $\alpha$ scales the power radiated by the array. The elements of $\mathbf{H}$ are given by the complex channel coefficients between each antenna and each user, as illustrated in Figure 2. The coefficients are given by:

$$
h_{k, n}=A_{k, n} e^{j \frac{2 \pi}{\lambda_{0}}\left(r_{k}-p_{n} \sin \theta_{k}\right)}
$$

where $p_{n}$ denotes the position of antenna element $n$ on the x-axis, $\theta_{k}$ the angle of user $k$ with respect to the base station, and $r_{k}$ the distance of user $k$ to the base station. $\lambda_{0}$ is the wavelength at center frequency $f_{0}$. Amplitude $A_{k, n}$ represents the intensity of the channel component. This is given by:

$$
A_{k, n}=10^{\frac{G_{\mathrm{TX}, n}\left(\theta_{k}\right)+G_{\mathrm{RX}}-P_{\mathrm{CI}, \mathrm{dB}, k}}{20}}
$$

where $G_{\mathrm{TX}}\left(\theta_{k}\right)$ is the gain function in $\mathrm{dB}$ of element $n$ in the array in the direction of user $k$ and $G_{\mathrm{RX}}$ is the gain pattern at the receiver end in $\mathrm{dB} . P_{\mathrm{CI}, \mathrm{dB}, k}$ represents the path loss in $\mathrm{dB}$ towards user $k$ according to the close-in (CI) path loss model [19]. This path loss model is given by:

$$
P_{\mathrm{CI}, \mathrm{dB}, k}\left(f_{0}, r_{k}\right)=P_{\mathrm{fspl}, \mathrm{dB}}\left(f_{0}, 1 m\right)+10 m \log _{10}\left(r_{k}\right)+\chi_{\sigma}^{\mathrm{CI}}
$$

with $m$ the path loss exponent and $\chi_{\sigma}^{\mathrm{CI}}$ describing the shadow fading term in $\mathrm{dB} . P_{\mathrm{fspl}, \mathrm{dB}}\left(f_{0}, 1 \mathrm{~m}\right)$ is the free-space path loss in $\mathrm{dB}$ at a distance of $1 \mathrm{~m}$ from the base station, which is found using:

$$
P_{\mathrm{fspl}, \mathrm{dB}}\left(f_{0}, 1 m\right)=20 \log _{10}\left(\frac{4 \pi f_{0}}{c_{0}}\right)
$$

with $c_{0}$ the speed of light in a vacuum. The values $m$ and $\chi_{\sigma}^{C I}$ for different scenarios are given in Table 1. 


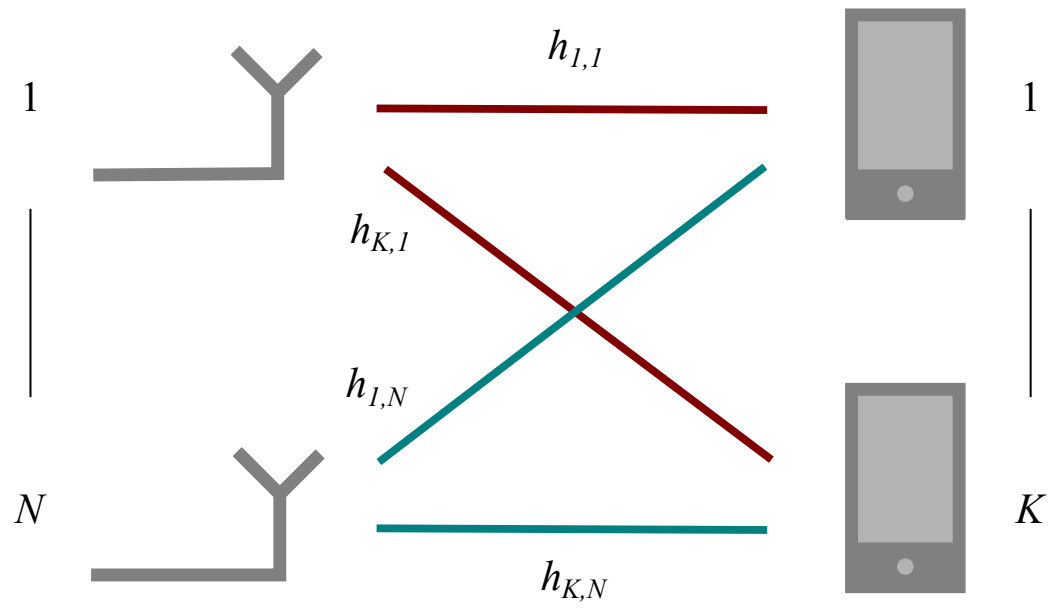

Figure 2. Channel matrix $\mathbf{H} \in \mathbb{C}^{K \times N}$ is constructed by finding the complex channel coefficients between each antenna and each user, for $N$ antennas and $K$ users.

Table 1. CI path loss model parameters for different Urban Macro (UMa), Urban Micro (UMi), line of sight (LoS) and non line of sight (nLoS) scenarios [19].

\begin{tabular}{cc}
\hline Scenario & CI Parameters \\
\hline UMa-LoS & $m=2.00, \chi_{\sigma}^{\mathrm{CI}}=4.1 \mathrm{~dB}$ \\
UMa-nLoS & $m=3.00, \chi_{\sigma}^{\mathrm{CI}}=6.8 \mathrm{~dB}$ \\
UMi-Street Canyon-LoS & $m=1.98, \chi_{\sigma}^{\mathrm{CI}}=3.1 \mathrm{~dB}$ \\
UMi-Street Canyon-nLoS & $m=3.19, \chi_{\sigma}^{\mathrm{CI}}=8.2 \mathrm{~dB}$ \\
UMi-Open Square-LoS & $m=1.85, \chi_{\sigma}^{\mathrm{CI}}=4.2 \mathrm{~dB}$ \\
UMi-Open Square-nLoS & $m=2.89, \chi_{\sigma}^{\mathrm{CI}}=7.1 \mathrm{~dB}$ \\
\hline
\end{tabular}

The ZF pre-coding matrix $\mathbf{W}$ can now be found using:

$$
\mathbf{W}=\mathbf{H}^{\dagger}\left(\mathbf{H H}^{\dagger}\right)^{-1}
$$

where + denotes the Hermitian transpose. By scaling $\mathbf{W}$ by a factor $\alpha$ as in:

$$
\alpha=\sqrt{\frac{P_{\max }}{\max \sum_{k=1}^{K}\left|w_{k, n}\right|^{2}}}
$$

for $1 \leq n \leq N$, it is ensured that the power per antenna element does not exceed a specified maximum per-antenna power $P_{\max }$. Thus, the model applies ZF under a per-antenna power constraint.

\subsection{Figure of Merit}

By applying the model as described in Section 2.1, the received power and interference at each user can be found. The CINR of each user $\left(\mathrm{CINR}_{k}\right)$ can be calculated using:

$$
\mathrm{CINR}_{k}=\frac{C_{k}}{I_{k}+N_{0} B}
$$

where the carrier power at user $k, C_{k}$, can be deduced from the diagonal elements of $\alpha \mathbf{H W}$, as in:

$$
C_{k}=\left|[\alpha \mathbf{H W}]_{k, k}\right|^{2} \text {. }
$$

The noise spectral density is $N_{0}=k_{B} T_{0}=-174 \mathrm{dBm} / \mathrm{Hz}$, with $k_{B}$ the Boltzmann constant and $T_{0}$ equal to $293 \mathrm{~K}$. The interference at user $k, I_{k}$, in case of ZF and perfect CSI, is always zero. This means the CINR is equal to the carrier-to-noise ratio (CNR). As such, (8) becomes: 


$$
\mathrm{CINR}_{k}=\mathrm{CNR}_{k}=\frac{|\alpha \mathbf{H W}(k, k)|^{2}}{N_{0} B} .
$$

With this, the CNR can be found for any $N$ and any $K \leq N / 2$, for any array composition and for any set of positions of the $K$ users inside the sector. The arrays under test can be compared to each other by using a statistical approach, similar to what was implemented in [20]. The $K$ users are distributed randomly in the sector many times, (e.g., 100,000 or 1,000,000 times), and the received CNR at each user is found for each case. The probability that the CNR is lower than a certain level can be found by using the cumulative distribution function (CDF).

\subsection{Simulation Parameters}

The center frequency $f_{0}$ was set to $28.5 \mathrm{GHz}$, and the $B$ was set to $500 \mathrm{MHz}$. The chosen path loss model in this paper was the Urban Micro (UMi) Street-Canyon (SC) LoS model (UMi-SC-LoS). As such, $m=1.98$ and $\chi_{\sigma}^{\mathrm{CI}}=3.1 \mathrm{~dB}$. The users were randomly positioned inside the $120^{\circ}$ sector, $r_{\min }=10 \mathrm{~m}$, and $r_{\max }=100 \mathrm{~m}$. The chosen $r_{\max }$ corresponds to the maximum distance of a user to the base station in an UMi scenario, as suggested in [19]. The user terminals were assumed to be at the same height as the base stations so that no vertical scanning was necessary. For $G_{\mathrm{TX}}$, we assumed a gain function that had a flat-top pattern, with a gain of $10 \mathrm{dBi}$ between $\theta= \pm 60^{\circ}$, and no radiation elsewhere. The minimum spacing between the centers of the array elements was $2 \lambda_{0}$, and antenna elements with a size of $2 \lambda_{0}$ were used. These settings were based on the horn antenna design that we use during the measurements in Section 5. The large antenna spacing means that this is a sparse array.

For the receiver antenna, we assumed it was isotropic and the polarization was perfectly matched. As such, $G_{R x}=0 \mathrm{dBi}$. The channel state information (CSI) was assumed to be perfect, and no scheduler was used.

\section{Regular and Irregular Arrays}

\subsection{Regular Arrays}

Four different regularly spaced arrays were now simulated using the approach and parameters as defined in Section 2. The arrays under test included two arrays with eight elements, with $0.5 \lambda_{0}$ (dense) and with 3.0 $\lambda_{0}$ (sparse) inter-element spacing, and two arrays with sixteen elements, also with $0.5 \lambda_{0}$ and $3.0 \lambda_{0}$ spacing. The $0.5 \lambda_{0}$ array could not be constructed because the element size was set to $2 \lambda_{0}$, meaning the elements would overlap. Nevertheless, it is interesting to investigate whether the dense arrays would perform better than the sparse ones, even though they cannot be constructed in reality. For this test, $K=2$ for all cases and the number of iterations $I$ was 1,000,000. The results are shown in Figure 3 . This figure shows the CDF of the CNR at the receiver. It can be seen that for the dense $N=8$ array, the outage probability was $3.3 \%$. For the sparse $N=8$ array, this probability was $2.9 \%$. Similarly, for the dense $N=16$ array, the outage probability was $0.88 \%$, and for the sparse $N=16$ array, this probability was $0.75 \%$.

The conclusion that can be drawn from this simulation is that the sparse arrays performed slightly better than the dense arrays in this scenario, for both numbers of elements. This seems counterintuitive at first, given that the sparse array had multiple grating lobes inside the FoV. Whenever one of the users was in or close to the a grating lobe of the beam directed towards another user, an outage occurred. It should be noted that every user experienced an outage whenever this happened, because the matrix $\mathbf{H H}^{\dagger}$ in (6) became singular. The reason the dense arrays did not perform better than the sparse arrays was because their main beams were much wider than those of the sparse ones. This means users were more likely to be close to another user's main beam, which also caused an outage. These two effects tended to cancel each other, but not entirely. A similar conclusion was reached in [15], where it was shown that sparse arrays led to a lower probability that the streams of two users became correlated. In [16], it was shown that an improvement of 
the stability of the inversion in (6) occurred when the antenna elements were spaced in a sparse regular fashion. This was also in line with the result that was found here, since the outages occurred whenever the matrix $\mathbf{H H}^{\dagger}$ became singular (i.e., the matrix was unstable).

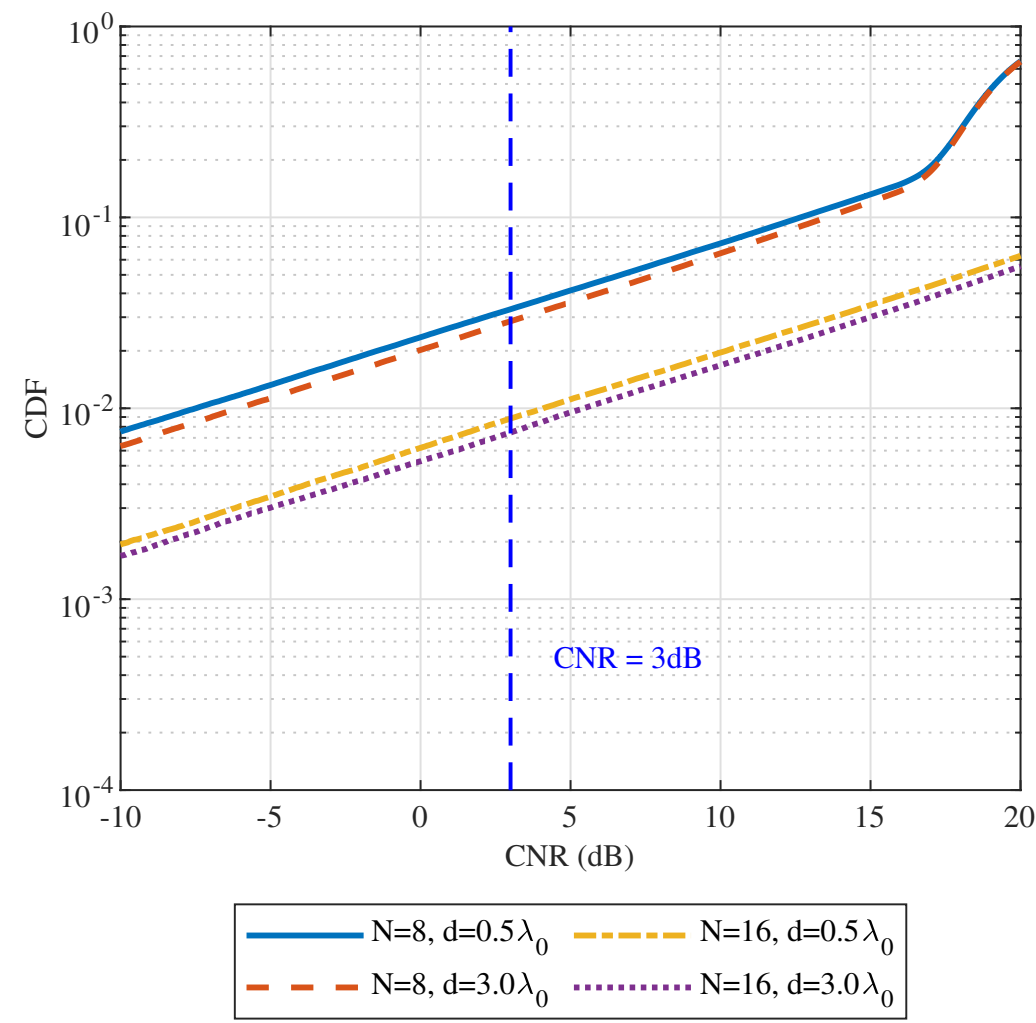

Figure 3. Cumulative distribution function (CDF) of the carrier-to-noise ratio (CNR) for different regular arrays. It is shown that there is only a small difference between dense and sparse arrays, for the cases where $N=8$ and $N=16 . K=2$ for both cases.

\subsection{Random Arrays}

Now, two sparse arrays with irregular inter-element spacings were generated, one with $N=8$ and one with $N=16$. These were then compared to the sparse regular arrays from Figure 3. The arrays were randomly generated with the constraints that the spacing between two consecutive elements was always at least $2 \lambda_{0}$ and that the total array size corresponded to the array sizes of the regular arrays mentioned before. As such, the size of the $N=8$ array was $21 \lambda_{0}$ and the size of the $N=16$ array was $42 \lambda_{0}$. The results are given in Figure 4.

The improvement in terms of the outage probability was significant. For the $N=8$ array, the outage probability dropped from $3.3 \%$ to $0.52 \%$, and for the $N=16$ array, from $0.75 \%$ to $0.16 \%$. The performance of the random eight-element array even exceeded that of the regular sixteen-element array for any $C N R<9.3$. This showed that similar or better performance can be achieved with a reduced number of antenna elements in this type of scenario when the antenna elements are placed irregularly, effectively decreasing the cost of such an antenna array. Another way of looking at this result is that the users in the sector had a higher probability of experiencing a high CNR, which means they could enjoy a high data rate more often when an irregular array was employed instead of a regular one. The array parameters and the corresponding results are summarized in Table 2. 


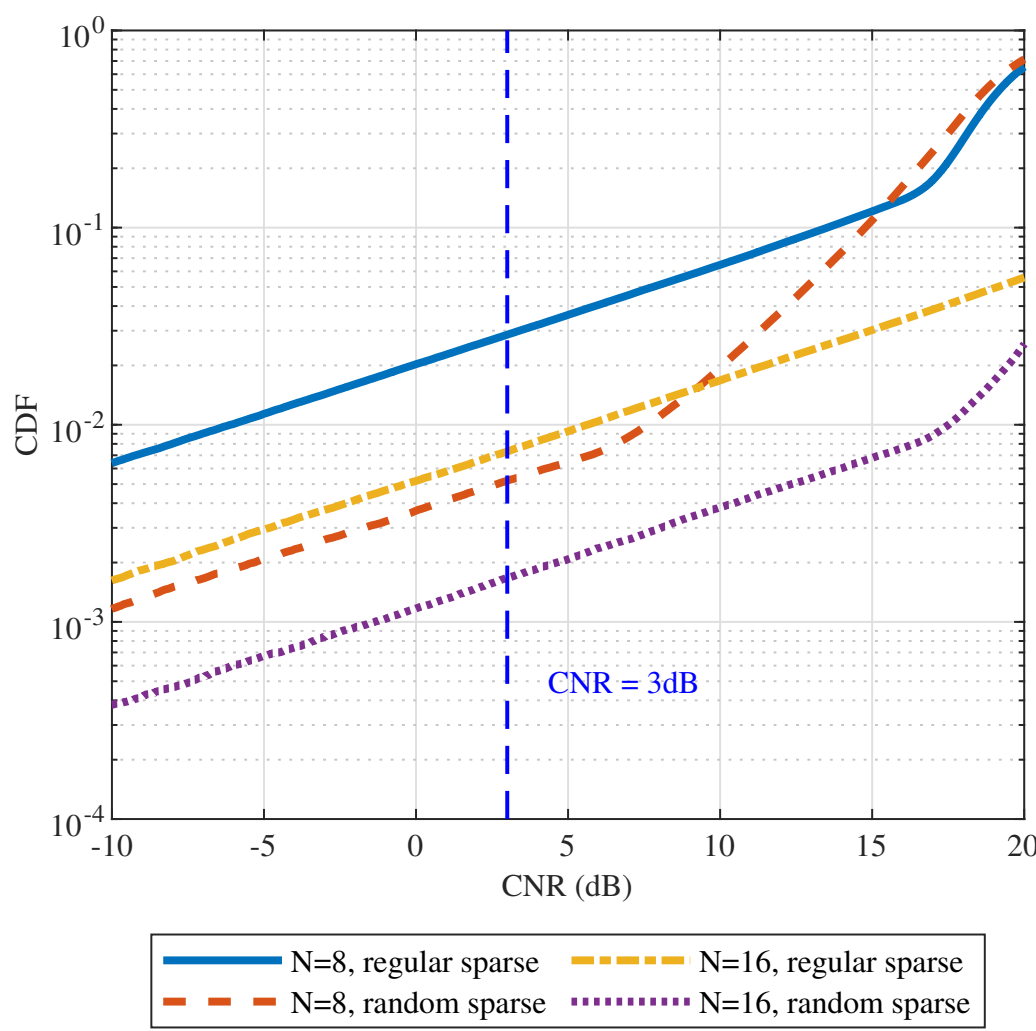

Figure 4. Cumulative distribution function of the CNR for regular and irregular (random) arrays. The improvement in terms of outage probability is substantial when considering random arrays, for both cases where $N=8$ and $N=16$. The sizes are $21 \lambda_{0}$ for both $N=8$ arrays and $42 \lambda_{0}$ for both $N=16$ arrays. $K=2$ for both cases.

Table 2. Array parameters and calculated outage probability.

\begin{tabular}{ccccc}
\hline Array Type & $\boldsymbol{N}$ & Inter-Element Spacing & Total Size & Outage Probability \\
\hline Regular dense & 8 & $0.50 \lambda_{0}, 5.3 \mathrm{~mm}$ & $4 \lambda_{0}, 42 \mathrm{~mm}$ & $3.3 \%$ \\
Regular sparse & 8 & $3.0 \lambda_{0}, 31.6 \mathrm{~mm}$ & $24 \lambda_{0}, 253 \mathrm{~mm}$ & $2.9 \%$ \\
Regular dense & 16 & $0.50 \lambda_{0}, 5.3 \mathrm{~mm}$ & $8 \lambda_{0}, 84 \mathrm{~mm}$ & $0.88 \%$ \\
Regular sparse & 16 & $3.0 \lambda_{0}, 31.6 \mathrm{~mm}$ & $48 \lambda_{0}, 506 \mathrm{~mm}$ & $0.75 \%$ \\
Irregular sparse & 8 & $\geq 2.0 \lambda_{0}, \geq 21.1 \mathrm{~mm}$ & $21 \lambda_{0}, 221 \mathrm{~mm}$ & $0.52 \%$ \\
Irregular sparse & 16 & $\geq 2.0 \lambda_{0}, \geq 21.1 \mathrm{~mm}$ & $42 \lambda_{0}, 442 \mathrm{~mm}$ & $0.16 \%$ \\
\hline
\end{tabular}

From this analysis, it became clear that better performance was possible when the elements were placed irregularly. Until now, the irregular arrays were generated randomly. This means that each time an array was generated with a new set of random numbers, the performance slightly changed. However, the variance between arrays that were generated randomly was quite small, and the performance was not highly dependent on the positions of the elements, as long as they remained random.

To show this, 800 random $N=8$ arrays were generated with a total size of $21 \lambda_{0}$ and the outage probability was found for each array. The results are plotted in a histogram in Figure 5. It shows how often an array was generated corresponding to a certain outage probability. The mean of the outage probabilities was $0.59 \%$. From the figure, it can be seen that slightly better arrays than the mean were possible: the best array gave a $0.48 \%$ outage probability. The worst one that was found was at $1.1 \%$. This result is interesting, as it was already shown that a regular sparse array in the same scenario had an outage probability of $2.9 \%$, higher than any of the random arrays simulated here. 


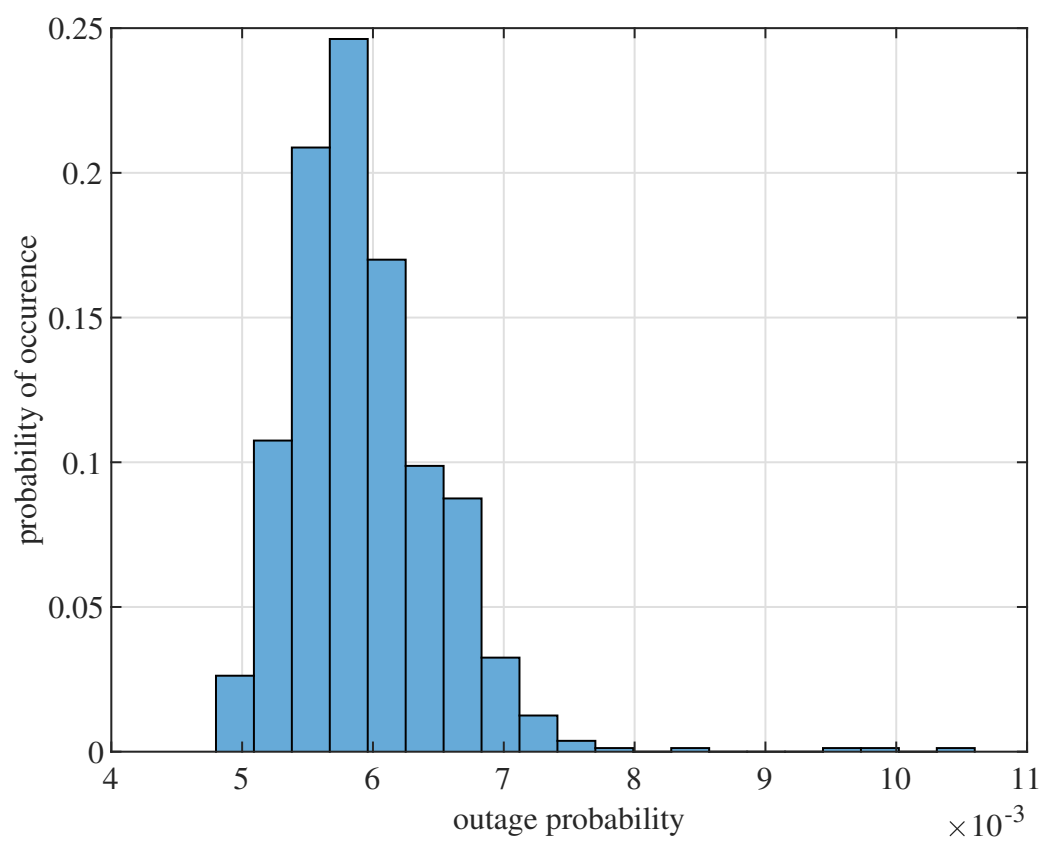

Figure 5. Histogram of the outage probability for 800 random arrays shows that the performance of the array is not very sensitive to the element positions, as long as they are generated randomly.

\section{Configuration Optimization}

In the previous section, it was shown that a random distribution of the antenna elements led to a better performance than regular configurations. The question that remains is how the element positions can be optimized. Using the method of generating many arrays and selecting based on the CDF is possible, but this is a very slow optimization, especially when a larger number of antennas is considered. Additionally, generating the optimum array during a random search could be unlikely, similar to how it is unlikely that the regular array is generated through random placement.

In this section, some existing metrics related to the SLL and measures of "irregularity" are explored to see if there was a correlation with the outage probability. Here also, eight antennas and two users were considered, with an array size of $21 \lambda_{0}$.

\subsection{Side Lobe Level and Outage Probability}

In order to investigate if there was any correlation between the outage probability and the SLL of the array, 800 random arrays were generated, and the simulation was run again. The outage probability was found, and the SLL was calculated for each array when considering a uniform excitation. These quantities are plotted in a scatter plot in Figure 6.

From this figure, it became clear that there was an insignificant correlation between the SLL and the outage probability. It seems that optimizing for the SLL did not necessarily give the optimum array in terms of the outage probability.

\subsection{Number of Independent Baselines and Outage Probability}

An array with $N$ elements contains $N(N-1) / 2$ pairs of antennas. One can list the distances between the antennas in each pair. We define this distance a "baseline". For example, for a regularly spaced $N=4$ array, with unit spacing $u$, the list of baselines is $\left[d_{u}(\times 3), 2 d_{u}(\times 2), 3 d_{u}\right]$. This shows some baselines are sampled two or more times. In total, only three independent baselines $\left(N_{\text {ind }}\right)$ are sampled, out of the six total baselines. By positioning the elements in a specific way, $N_{\text {ind }}$ can be maximized. The method of maximizing $N_{\text {ind }}$ is relevant in the application of radio-astronomy, but it is interesting to 
investigate if this technique may be applicable in the considered scenario. Consider the case where the largest baseline $b$, equal to the array size, is given as:

$$
b=d_{u} \cdot \frac{N(N-1)}{2}
$$

then for an $N=4$ array, $b=6 d_{u}$. Then, if the four elements of an $N=4$ array are placed at the positions $x=\left[0 d_{u}, 1 d_{u}, 4 d_{u}, 6 d_{u}\right]$, the list of baselines becomes $\left[d_{u}, 2 d_{u}, 3 d_{u}, 4 d_{u}, 5 d_{u}, 6 d_{u}\right]$, giving $N_{\text {ind }}=6$.

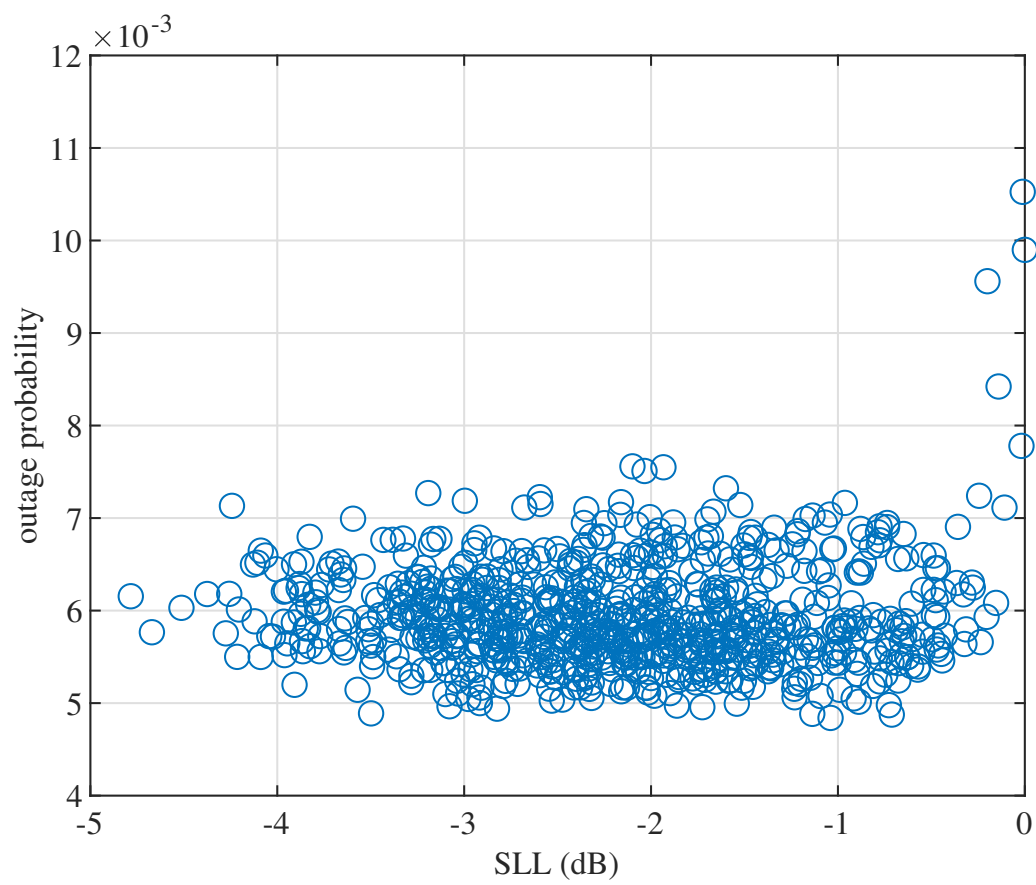

Figure 6. Scatter plot of 800 different random arrays showing only a small correlation between the SLL and the outage probability. The SLL is found when the array is uniformly excited.

Now, consider the case where eight antenna elements were placed randomly, but the positions were limited to multiples of $0.5 \lambda_{0}$, while the minimum distance between two elements was still 2.0 $\lambda_{0}$. Another 800 arrays were generated, and the simulation was run. $N_{\text {ind }}$ is plotted against the outage probability in Figure 7 . The figure shows two distinct regions, one where $N_{\text {ind }}$ is low and the outage probability is high and one where the outage probability is low, with $N_{\text {ind }}$ ranging between 12 and 25 .

The reason the outage probability was high for some samples was because for those samples, the elements were spaced at regular intervals. This increased the outage probability, similar to what was found in Section 3. It appears that the number of independent baselines is not directly a good measure of irregularity, otherwise we would not expect to have these two separate regions.

The reason outage probabilities greater than 1.1\% were not found in Figure 6 was that it was much more likely that arrays with high regularity were found when the positions were always a multiple of $0.5 \lambda_{0}$. In Figure 6, however, this was not the case, and the elements could have any position.

In the end, one can conclude that if an array has a high $N_{\text {ind }}$, it also has a low outage probability. However, it is also clear that optimizing for $N_{\text {ind }}$ does not necessarily result in the optimum array configuration. 


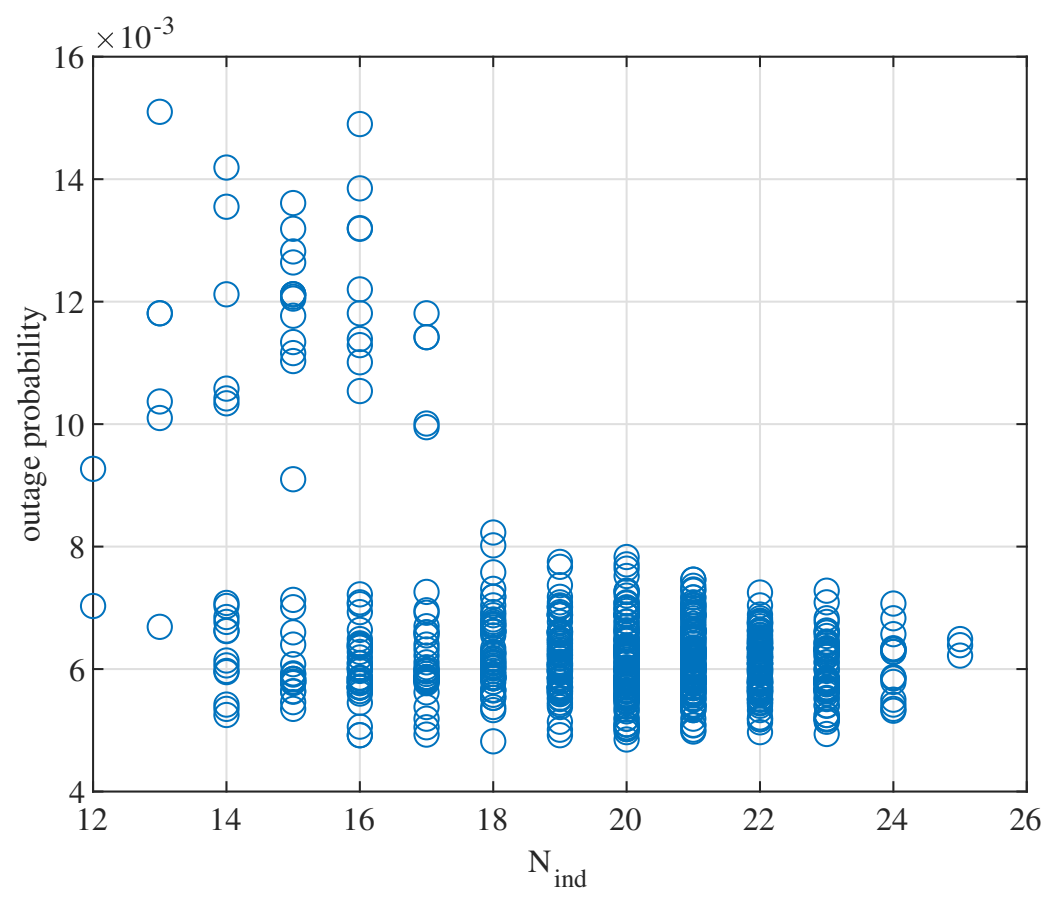

Figure 7. Scatter plot of 800 different random arrays showing the number of independent baselines in the linear array compared to its outage probability.

\subsection{Minimum Redundancy Linear Array}

This next type of array was based on the concept of the minimum redundancy linear array (MRA) [21]. In an MRA, the redundancy ratio $R$ defined as:

$$
R=\frac{N(N-1)}{2} \cdot \frac{1}{N_{\max }}
$$

is minimized. In this, $N_{\max }$ denotes the greatest multiple of the unit spacing such that all multiples of the unit spacing $\leq N_{\max }$ are present in the array. For example, let us consider the regularly spaced $N=4$ array, with unit spacing $d_{u}$. The list of baselines is $\left[d_{u} \times 3,2 d_{u} \times 2,3 d_{u}\right]$. It follows that $N_{\max }=3$ and that $R=2$.

Consider again the case where the elements are positioned at $x=\left[0 d_{u}, 1 d_{u}, 4 d_{u}, 6 d_{u}\right]$. The list of baselines is $\left[d_{u}, 2 d_{u}, 3 d_{u}, 4 d_{u}, 5 d_{u}, 6 d_{u}\right]$, which gives $N_{\max }=6$ and $R=1$. This means, according to the definition of an MRA, there is no redundancy in the array. Coincidentally, this is the largest linear array that can be formed with $R=1$ [21,22].

Now, applying this to the problem of this paper means the following:

1. The positions of the elements must be an integer multiple of the grid spacing. This was chosen to be $0.5 \lambda_{0}$;

2. The elements, having a size of $2.0 \lambda_{0}$, cannot overlap;

3. $b$ was set to a fixed $21 \lambda_{0}$.

The outer two elements were always $21 \lambda_{0}$ apart. Now, the remaining six elements were placed randomly 800 times with the given constraints, and the simulation was repeated. The resulting scatter plot is shown in Figure 8. It shows that there was no clear relation between the redundancy ratio $R$ and the outage probability, but it seems that the worst results were obtained for $R=14$. The lowest $R$ that was obtained in this experiment was 2.34. The most important observation from the figure is that optimizing $R$ does not necessarily optimize the outage probability. 


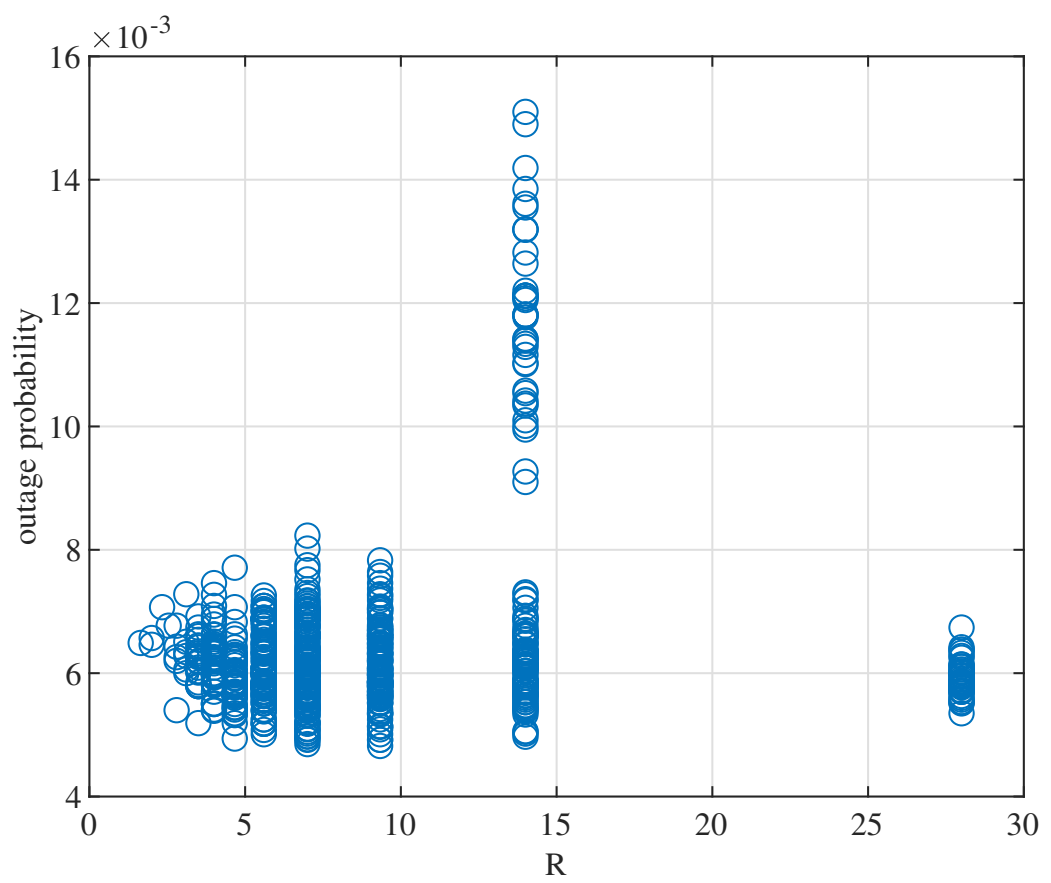

Figure 8. Scatter plot of 800 arrays showing the outage probability compared to the redundancy ratio $R$.

An important remark is that the MRA as defined in [21] only considers the possibility of elements being placed at multiples of the minimum unit spacing. With antennas having a size of $2.0 \lambda_{0}$, the minimum unit spacing would also be $2.0 \lambda_{0}$. If this is applied, it would mean that all elements are placed at a multiple of $2.0 \lambda_{0}$, and grating lobes would occur. For that reason, we chose to place elements at multiples of $0.5 \lambda_{0}$. However, this means the baselines $0.5 \lambda_{0}, 1.0 \lambda_{0}$, and $1.5 \lambda_{0}$ cannot be made, as the elements would overlap. This means $N_{\max }$ was calculated ignoring those baselines, and the list started at $2.0 \lambda_{0}$. As such, this implementation was strictly not in accordance with the definition of an MRA.

From this analysis, one can conclude that either optimizing for the SLL, $N_{\text {ind }}$, or $R$ does not necessarily give the optimum array in terms of the outage probability. However, it was also shown that any irregularly spaced array far outperforms a regularly spaced array, even when this irregularity is randomly generated. Furthermore, one can conclude that the performance of such irregularly spaced array is not impacted much by small variances in the element positions, as long as the array remains irregularly spaced. With this in mind, it was decided to create a prototype that allowed for the positions of the antenna elements to be re-configurable, such that regular and irregular configurations could be created and compared to each other.

\section{Prototype}

To show the sparse irregular array concept in practice, a re-configurable array was built using antenna elements with relatively large radiating apertures, which were realized using $3 \mathrm{D}$ printing of aluminum. The re-configurable array allowed both regular and irregular configurations to be tested.

\subsection{Single Element Design and Realization}

The element that was designed was based on the box-horn antenna [23]. This antenna type is a rectangular horn with a discontinuity at the feed. The discontinuity causes the generation of the $T E_{30}$ mode, next to the fundamental $T E_{10}$ mode supplied by the attached rectangular waveguide section. This results in multi-mode operation. This can be used to extend the scan range in the H-plane, while still operating as a normal horn antenna in the E-plane. When the H-plane is aligned with the horizontal plane, this element is suitable for use in base stations, because it has a wide scan range in the azimuth and a 
high gain. The antennas were fabricated by 3D printing of aluminum at Shapeways. The 3D printing process was selective laser melting (SLM). More details about the process and the exact composition of the material can be found on the company's website [24]. One of the fabricated antenna elements is shown in Figure 9. Printing with aluminum has the drawback of giving a higher surface roughness than other methods such as milling, though the surface roughness is acceptable at this frequency range. Small features such as holes or protrusions are not printed accurately however. This means the alignment of the coaxial connector was difficult. The SMK connector was mounted on the top of the waveguide section. The pin of the connector excites the waveguide. The dimensions are given in Appendix A.

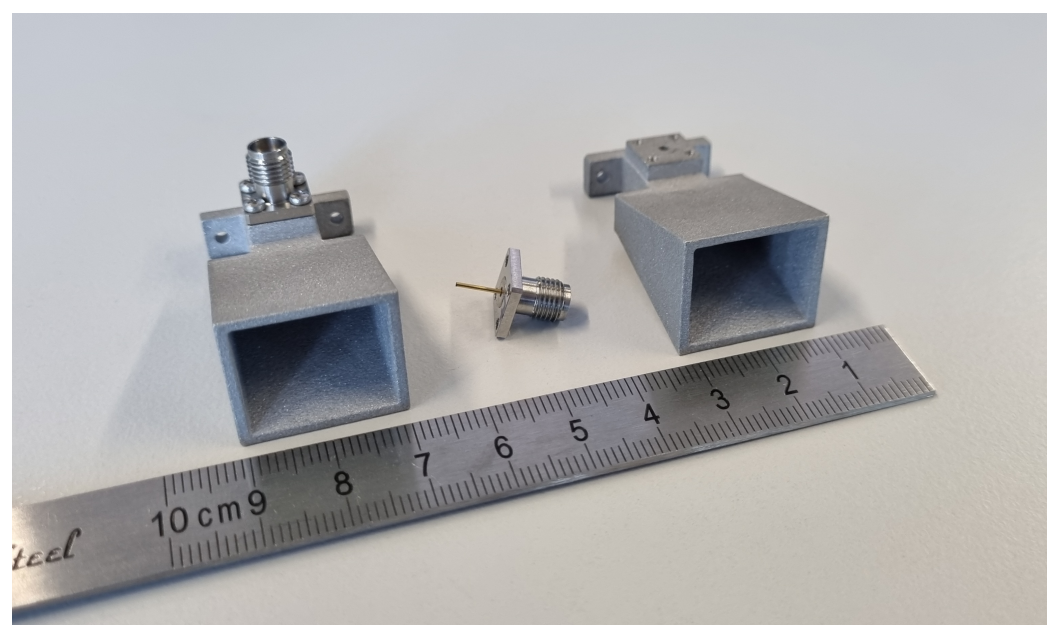

Figure 9. Two of the antenna elements produced by 3D printing of aluminum. One is shown with the mounted SMK connector and one without. The connector itself is also shown with the exposed pin. It can be seen that the surface roughness of the material is quite high.

\subsection{Array Realization}

The ground plane design is given in Appendix B. The ground plane had a slit running through the middle, which allowed the elements to be moved around. This allowed multiple configurations to be tested with the same antenna elements. The slit had a length of $338 \mathrm{~mm}\left(32.2 \lambda_{0}\right)$ and a width of $2 \mathrm{~mm}$.

The regular array configuration had eight elements and an inter-element spacing of $4.0 \lambda_{0}$. This setup is shown in Figure 10. The irregular array configuration also had eight elements and had an equal total size as the regular array. The positions of each element for the irregular array is given in Table 3. This positioning choice was random, as it was shown in Section 4 that it did not matter much where the elements were positioned exactly, as long as the spacing was irregular. The measurement setup for this irregular array is shown in Figure 11.

Table 3. Positions of the antenna elements of the irregular array.

\begin{tabular}{ccc}
\hline Element Number & Position $(\mathbf{m m})$ & Position $\left(\lambda_{\mathbf{0}}\right)$ \\
\hline 1 & 0 & 0 \\
2 & 26.3 & 2.50 \\
3 & 54.5 & 5.18 \\
4 & 81.6 & 7.75 \\
5 & 132.3 & 12.75 \\
6 & 169.6 & 16.11 \\
7 & 259.9 & 24.69 \\
8 & 294.7 & 28.00 \\
\hline
\end{tabular}




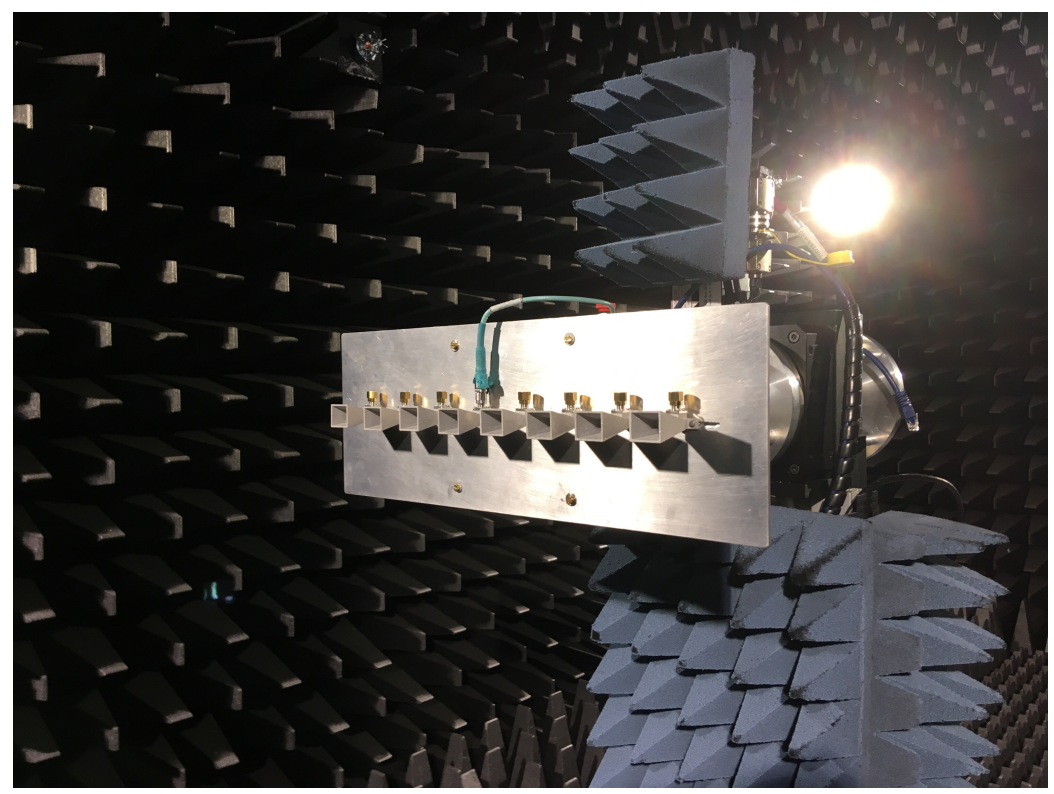

Figure 10. Picture of the array configured as a regular sparse array, mounted in the anechoic chamber.

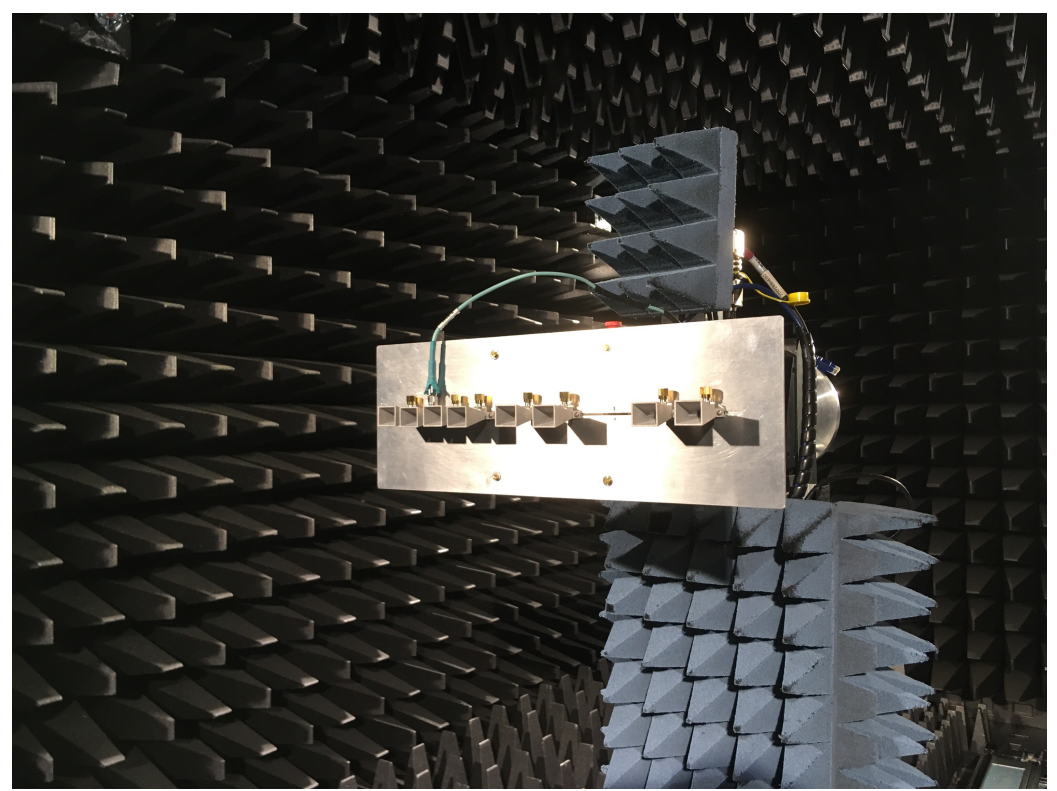

Figure 11. Picture of the array configured as an irregular sparse array, mounted in the anechoic chamber.

\subsection{Measurements}

The reflection coefficients were measured for each antenna element and compared to the simulated element. The simulation was performed with CST Microwave Studio. The results are shown in Figure 12. A spread was observed between the elements. It was clear that misalignment of the pin led to changes in the $S_{11}$. Regardless, $\left|S_{11}\right|$ was below $-10 \mathrm{~dB}$ from $26 \mathrm{GHz}$ up to about $38 \mathrm{GHz}$, which was adequate.

In order to evaluate the mutual coupling between elements of the array, two elements were placed on the ground plane at three different values for the inter-element spacing $d$, namely $2.0 \lambda_{0}, 3.0 \lambda_{0}$, and $4.0 \lambda_{0}$. The $S_{21}$ coefficient was measured. The results are shown in Figure 13. The same was done in the simulation assuming an infinitely large ground plane. The measurement gave lower values than the simulation. Regardless, the measured $S_{21}$ parameter was well below $-45 \mathrm{~dB}$ at $28.5 \mathrm{GHz}$ for all spacings. From this, it became clear that the mutual coupling was negligible in a situation with such antenna elements at large inter-element spacings. 


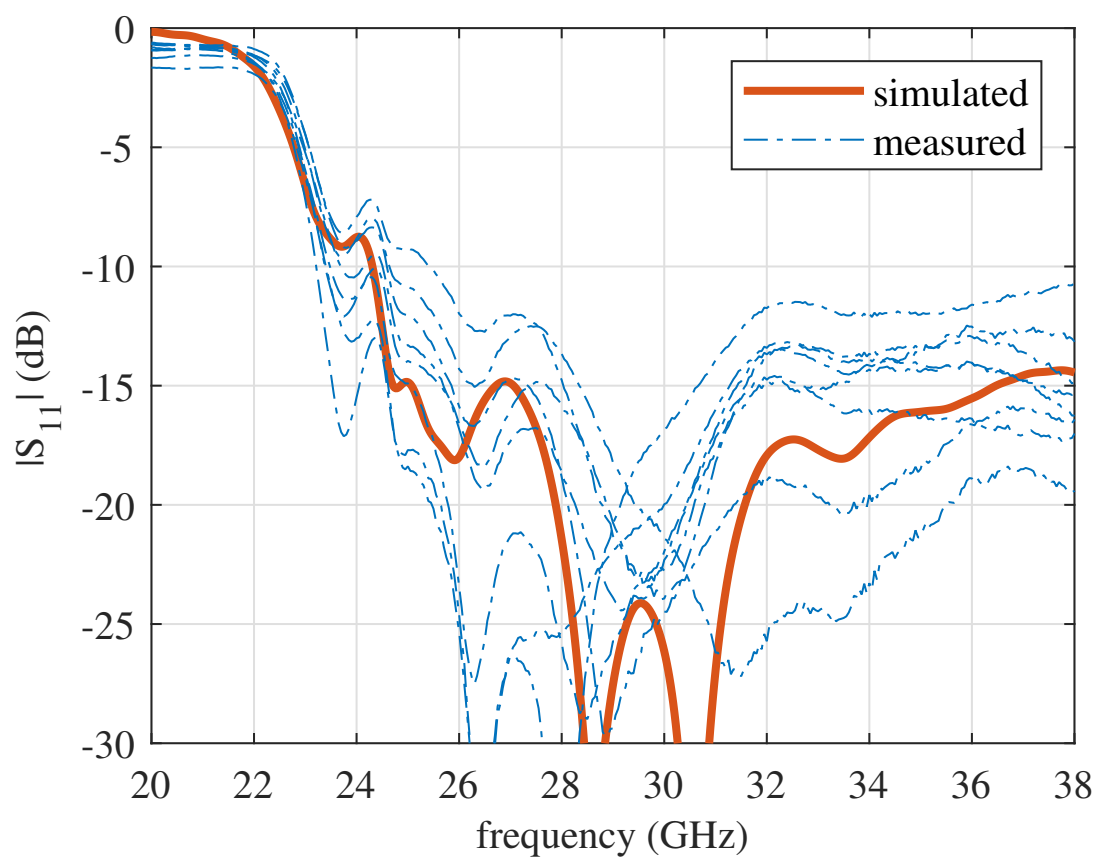

Figure 12. Measured reflection coefficient of each antenna element compared to the simulated reflection coefficient. There is a spread between the elements caused by misalignment of the excitation pin. The least-performing element has a reflection coefficient of $-15.6 \mathrm{~dB}$ at $28.5 \mathrm{GHz}$, which is adequate for the experiment.

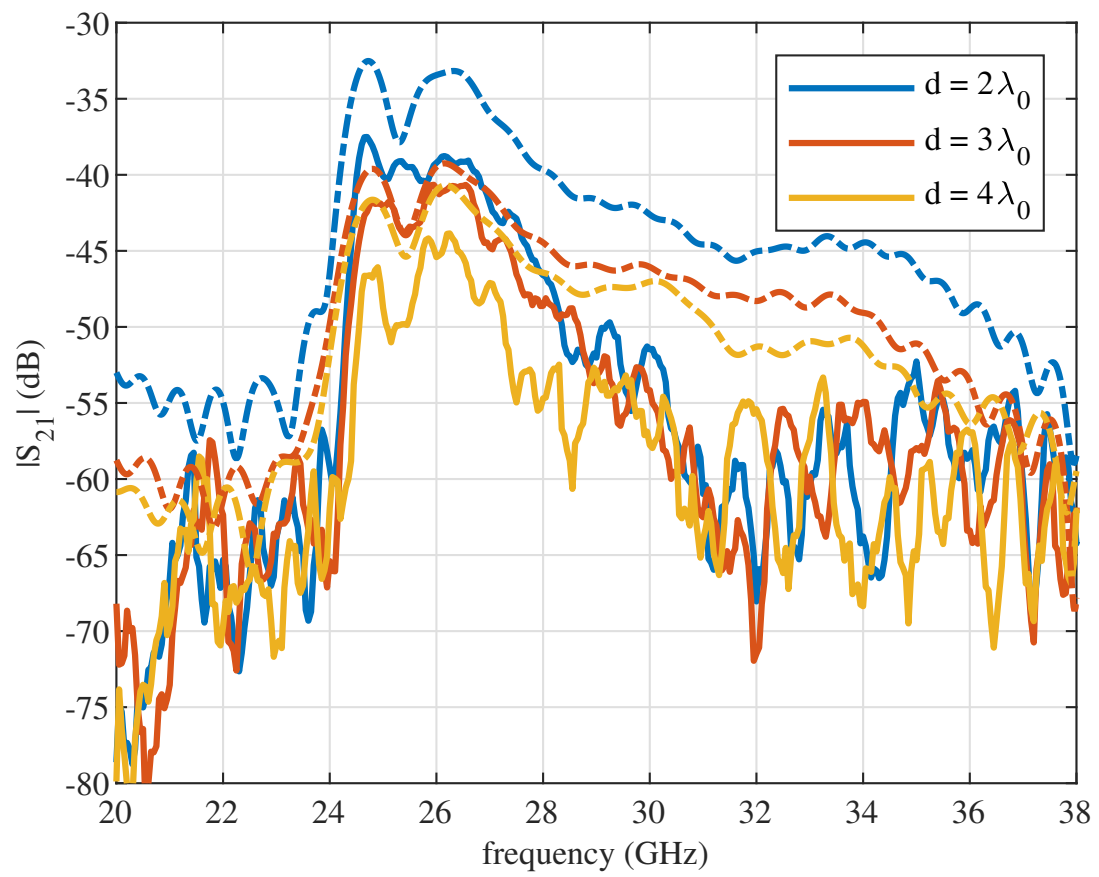

Figure 13. Measured (continuous lines) and simulated (dotted lines) $S_{21}$ coefficient for different values of the inter-element spacing $d$ show that the coupling at $28.5 \mathrm{GHz}$ is negligible.

The radiation patterns were then measured for each antenna when the array was configured in the regular layout as in Figure 10. When one antenna was excited, all others were terminated with a $50 \Omega$ load. A measurement of the gain was not taken, but in order to run the analysis, the gain of the elements must be known. We chose to normalize each pattern by setting the average gain between $\theta=-20^{\circ}$ and $\theta=20^{\circ}$ equal to the gain of the simulated antenna at $\theta=0^{\circ}$. This gave the gain patterns in Figure 14. A clear 
fringing pattern was present on the measured gain patterns, with a period $\theta_{p}$ of around $25^{\circ}$. By using:

$$
d_{f}=\frac{\lambda_{0}}{\sin \theta_{p}}
$$

the fringe spacing $d_{f}$ was found representing the distance between two interfering sources that were being measured. Considering $\theta_{p}=25^{\circ}$ and $\lambda_{0}=10.5 \mathrm{~mm}, d_{f}=24.8 \mathrm{~mm}$ was obtained. This almost corresponds to the physical distance between the walls of two neighboring elements, being $22.7 \mathrm{~mm}$. Thus, it can be concluded that waves were running across the ground and scattering off of neighboring elements, causing the fringing pattern.

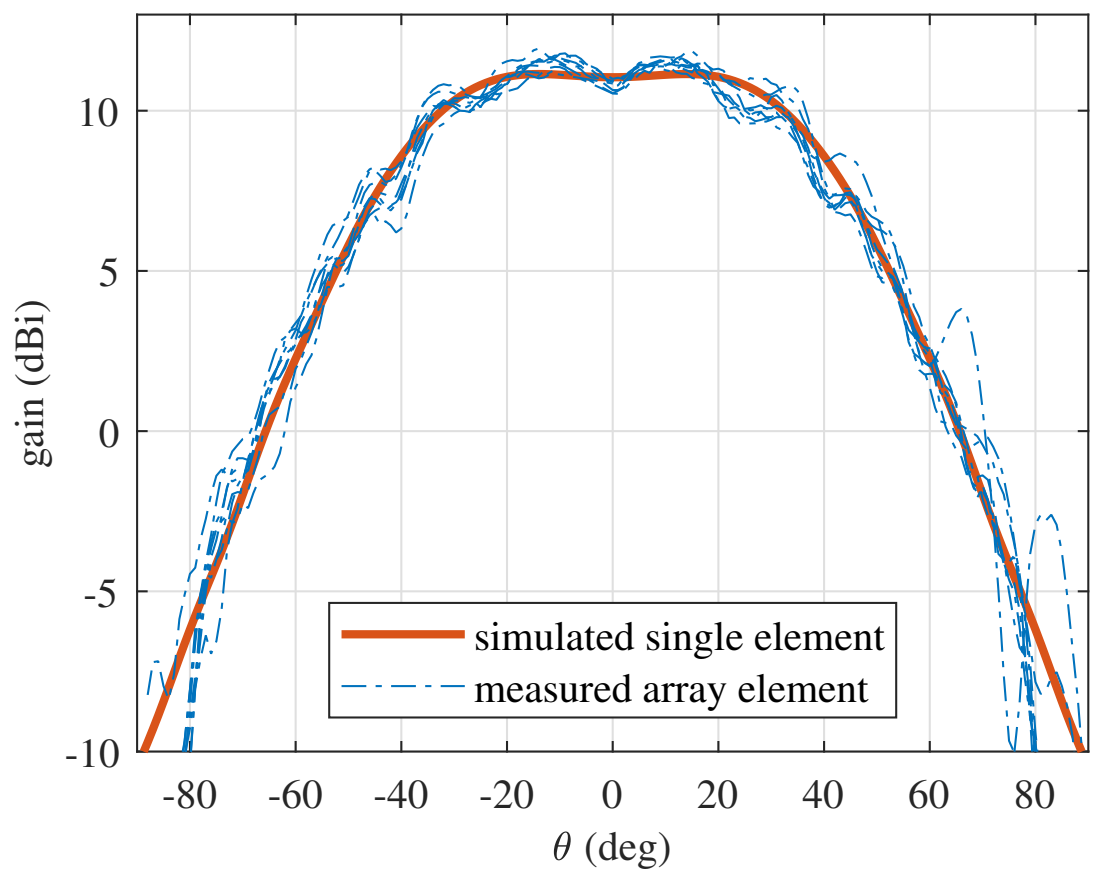

Figure 14. Measured radiation patterns of each antenna element when the array is configured as a regular array. The simulated single element, when considering an infinite ground plane, is overlaid. A measurement of the gain was not taken, but each pattern was normalized to the simulated gain at $\theta=0^{\circ}$.

The same measurement was conducted for the irregular array shown in Figure 11. The resulting patterns are shown in Figure 15. The only difference is that the fringe pattern became different for each measurement, because the spacings between the antenna elements were now also irregular.

\subsection{Outage Probability for Realized Prototype}

Knowing the gain patterns, the model in Section 2 can be improved by applying the patterns as $G_{T X}$ in (3). The simulated and measured gain patterns were considered for both the regular and the irregular array. The results from running the model are given in Figure 16. It became clear that when using real antenna elements, it holds true that an irregular array outperformed a regular one by a large margin. For the regular array with the measured patterns, the outage probability was $3.85 \%$; for the irregular array, it was a bit more than six-times lower, $0.64 \%$. The results with the flat-top patterns are also shown, showing that they provided a slightly better outage probability than the measured patterns. 


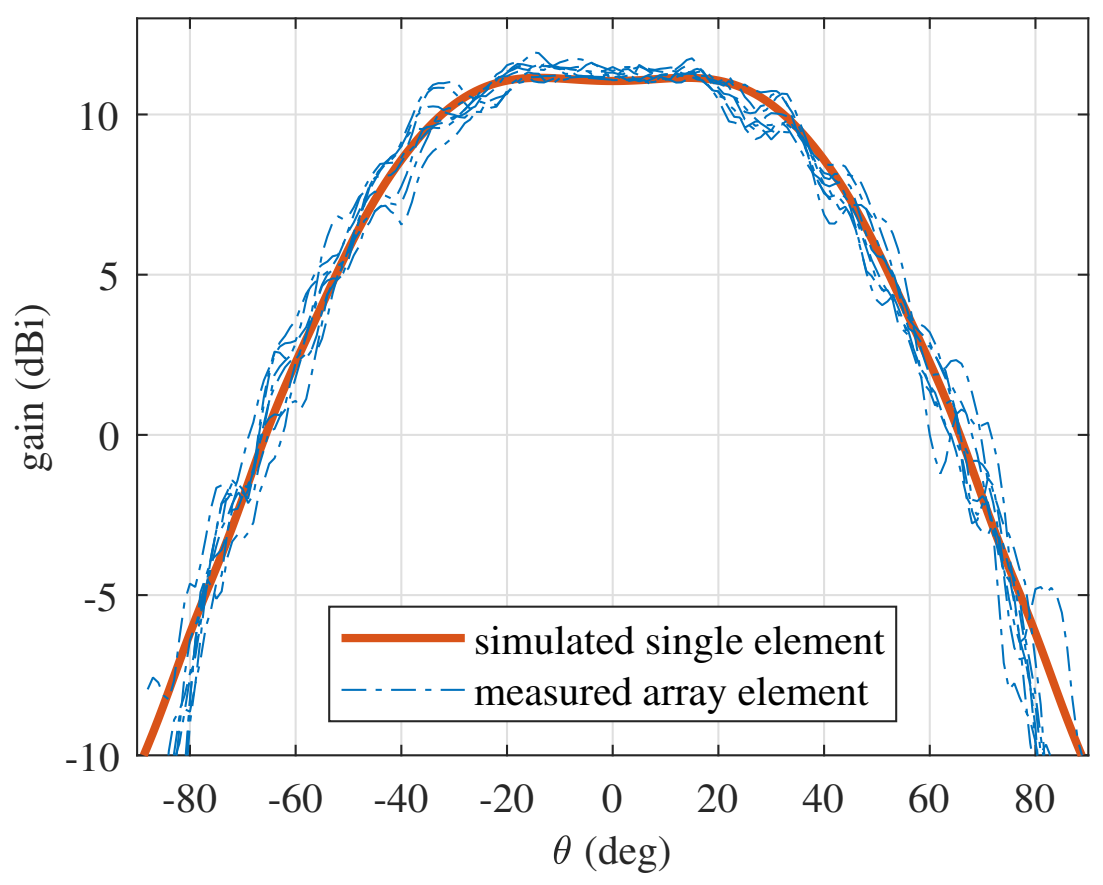

Figure 15. Measured radiation patterns of each antenna element when the array is configured as an irregular array. The simulated single element, when considering an infinite ground plane, is overlaid. A measurement of the gain was not taken, but each pattern was normalized to the simulated gain at $\theta=0^{\circ}$.

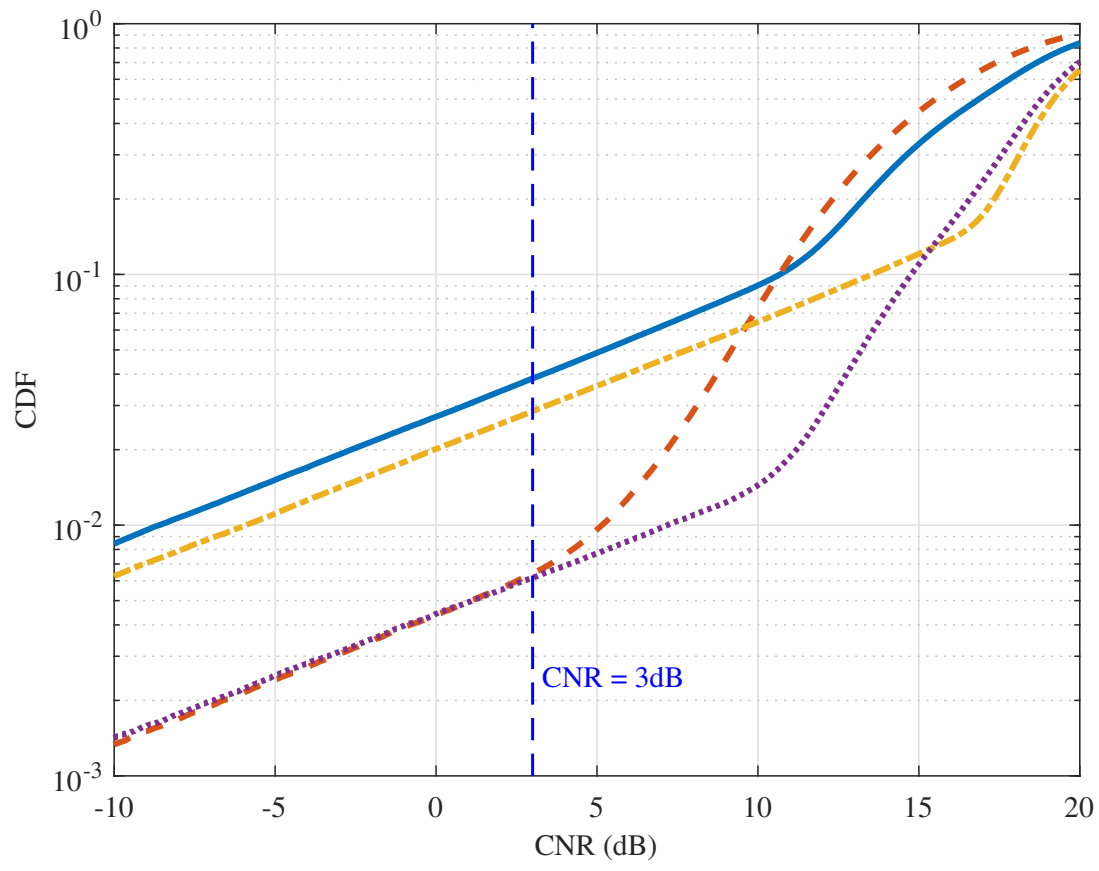

$$
\begin{gathered}
\hline- \text { regular sparse with measured patterns } \\
-E-\text { irregular sparse with measured patterns } \\
\ldots \ldots+\ldots \text { regular sparse with flat-top patterns } \\
\hline
\end{gathered}
$$

Figure 16. By applying the measured radiation patterns, the CDF of the CNR can be found. The irregular array still outperformed the regular array by a significant margin. This holds for both the simulated and measured patterns.

\section{Discussion and Recommendations for Future Work}

The results showed that an irregular array can outperform a regular array in a random LoS scenario by a large margin. This is promising, but there are many aspects to this 
problem that have not yet been investigated. For example, it would be interesting to see how imperfect CSI, increased mutual coupling, errors due to the quantization of the weights, scatterers, and NLoS conditions impact the performance of both types of arrays and if irregular arrays will still outperform regular ones taking these factors into account. Based on the evidence presented here and in related work, we expect that this is indeed the case.

Other than the technological advantage offered by sparse irregular arrays, the practicality of increased array sizes must also be considered. The array antenna that was built and shown here had inter-element spacings of up to eight wavelengths and had a total width of $30 \mathrm{~cm}$, which is quite large considering only eight antennas were used. In comparison, a classical dense array with eight antenna elements would only be around $4 \mathrm{~cm}$ to $5 \mathrm{~cm}$ wide at this frequency. An important design aspect of future $5 \mathrm{G}$ base stations is their physical appearance. They should look small and be able to blend in with the environment. In that sense, it is better to create smaller arrays rather than very large ones. This presents a trade-off between performance and appearance. For future work, it is interesting to explore this trade-off, for instance by starting the design procedure by determining a maximum acceptable array size and then investigating which type and size of antenna elements can be used and how the antenna elements should be positioned to arrive at an optimal performance given this constraint.

Furthermore, it may be difficult from an implementation perspective to build antenna arrays that are very sparsely and irregularly spaced. For future work, it is interesting to look into irregular configurations that are both relatively simple to implement and near optimal in terms of performance.

It was explained in Section 4 that the main cause of outages is that two or more users are located close to each other, causing the matrix in (6) to become singular. A solution to this issue, without applying a scheduler, is regularizing the inverse of the matrix, as proposed in [25]. Under this condition, it is no longer true that the interference is zero for all users. What the regularization implies is that users will start to receive more interference from each other as they move closer to each other, but the matrix will never become singular. In this case, an outage would occur when the signal-to-interference ratio becomes too high for co-located users. A further improvement of the ZF beamforming strategy described here is optimal beamforming [2]. For optimal beamforming, the weights are determined such that the optimal balance is found between maximizing the received signal power and minimizing the interference. For future work, it is relevant to investigate what the impact is on the system performance when employing this beamforming method rather than ZF.

The question that this work has not yet found a solution to is if there is an optimum configuration of the irregular array and if or how this can be found in a deterministic manner. This work attempted to answer this by investigating if optimizing the SLL, the number of independent baselines, or the redundancy ratio directly leads to a reduction in the outage probability, but this did not seem to be the case. Instead, the best performing antenna arrays were a result of randomizing the inter-element spacings.

As a general recommendation, we suggest further investigation of irregular sparse array configurations that balance good performance with manufacturability, size, and physical appearance.

\section{Conclusions}

A system-level study relating system performance to different array topologies was conducted. By investigating the probability that an outage occurs in a defined random LoS scenario, it was shown that irregular sparse arrays improved the system performance by decreasing the outage probability by a significant margin, even when a small number of antenna elements were used. Furthermore, it was shown that selecting array configurations based on metrics such as the side lobe level, the redundancy ratio, or the number of independent baselines did not necessarily lead to optimum array configurations. Instead, the best performances were obtained when the irregularity was randomly generated. To 
show this, a demonstrator array was designed and built with eight 3D-printed aluminum antenna elements. The array was re-configurable, which allowed multiple configurations to be tested. By taking measurements of the mutual coupling and the per-element radiation patterns, it was shown that irregular sparse arrays also outperformed regular sparse arrays when realistic antenna elements were used. With this demonstrator, the outage probability was reduced from $3.85 \%$ to $0.64 \%$ by moving from a sparse regularly spaced array to a sparse irregularly spaced array. This amounted to an improvement of a factor of six, without adding more antenna elements or increasing the total array size. This makes sparse irregular antenna arrays an important candidate for future mmWave base stations employing multi-user MIMO.

Author Contributions: Conceptualization, R.X.F.B.; methodology, R.X.F.B.; software, R.X.F.B.; validation, R.X.F.B. and M.N.J.; resources, M.V.I., A.B.S. and U.J.; writing—original draft preparation, R.X.F.B.; writing-review and editing, T.A.H.B., M.N.J., M.V.I., A.B.S. and U.J.; visualization, R.X.F.B.; supervision, T.A.H.B., M.V.I., A.B.S. and U.J.; project administration, A.B.S. All authors have read and agreed to the published version of the manuscript.

Funding: This research received no external funding.

Conflicts of Interest: The authors declare no conflict of interest.

\section{Abbreviations}

The following abbreviations are used in this manuscript:

EIRP effective isotropic radiated power

MU-MIMO multiple-user multiple-input multiple-output

ZF zero-forcing

FoV field of view

SLL side lobe level

CINR carrier-to-interference-plus-noise ratio

CNR carrier-to-noise ratio

LoS line of sight

nLoS non-line of sight

CDF cumulative distribution function

CSI channel state information

MRA minimum redundancy linear array

\section{Appendix A. Single Element Dimensions}
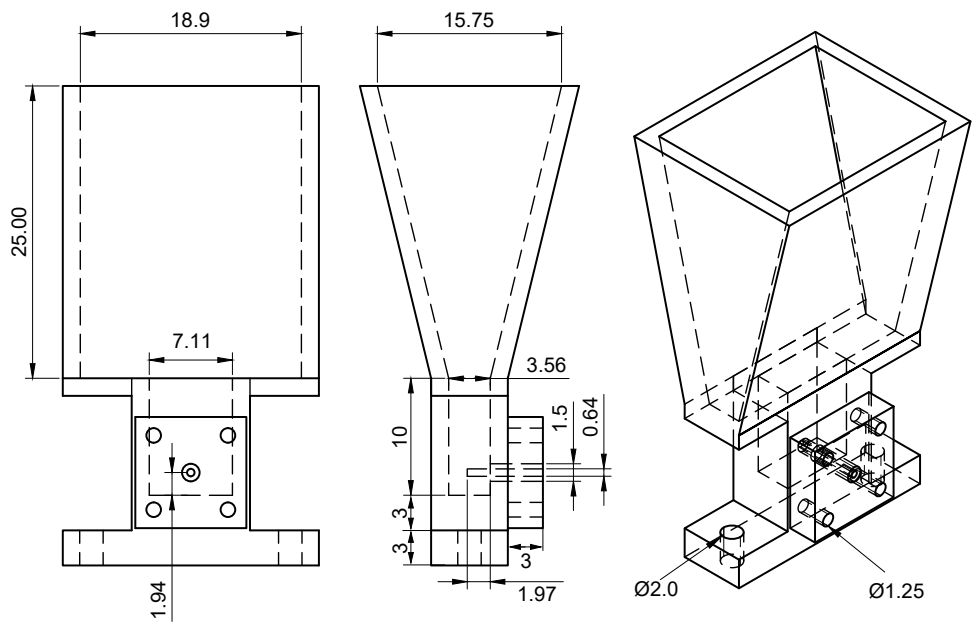

Figure A1. Dimensions of the antenna element. All dimensions are in $\mathrm{mm}$. The thickness of the walls is $1.5 \mathrm{~mm}$; the distance between the holes for the connector screws is $6.35 \mathrm{~mm}$, in a square pattern with the hole for the pin in the center. 


\section{Appendix B. Ground Plane Dimensions}

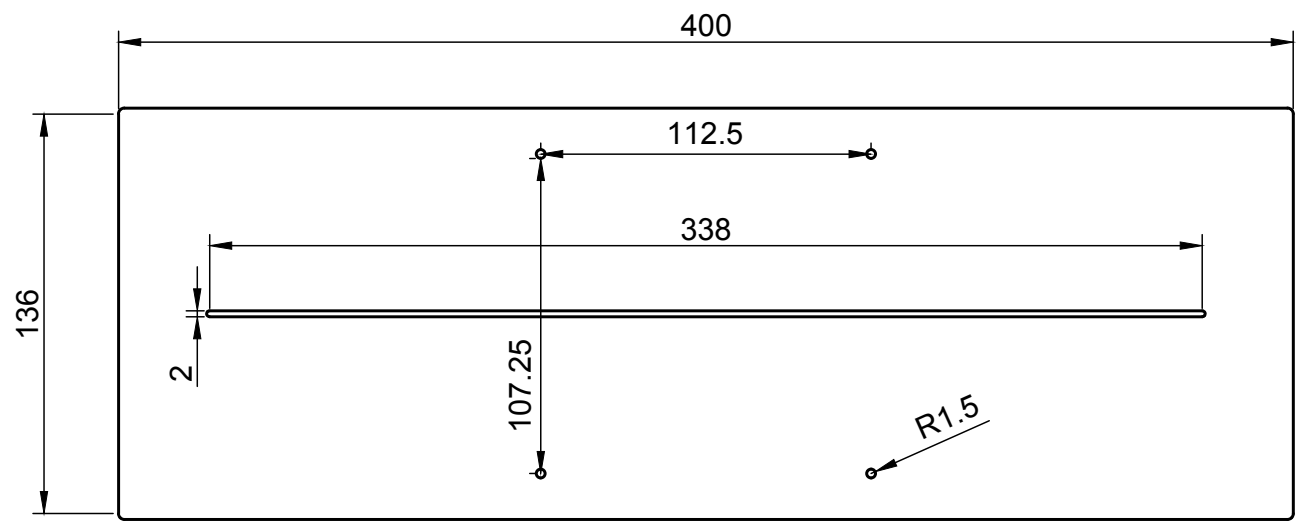

Figure A2. Dimensions of the ground plane. All dimensions are in $\mathrm{mm}$. The thickness of the plate is $3 \mathrm{~mm}$.

\section{References}

1. Ericsson. Ericsson Mobility Report. June 2021. Available online: https://www.ericsson.com/4a03c2/assets/local/reportspapers/mobility-report/documents/2021/june-2021-ericsson-mobility-report.pdf (accessed on 23 November 2021).

2. Björnson, E.; Bengtsson, M.; Ottersten, B. Optimal Multiuser Transmit Beamforming: A Difficult Problem with a Simple Solution Structure [Lecture Notes]. IEEE Signal Process. Mag. 2014, 31, 142-148. [CrossRef]

3. Albreem, M.A.; Juntti, M.; Shahabuddin, S. Massive MIMO Detection Techniques: A Survey. IEEE Commun. Surv. Tutor. 2019, 21,3109-3132. [CrossRef]

4. Rajatheva, N.; Atzeni, I.; Bjornson, E.; Bourdoux, A.; Buzzi, S.; Dore, J.B.; Erkucuk, S.; Fuentes, M.; Guan, K.; Hu, Y.; et al. White Paper on Broadband Connectivity in 6G. arXiv 2020, arXiv:eess.SP/2004.14247.

5. Yang, B.; Yu, Z.; Lan, J.; Zhang, R.; Zhou, J.; Hong, W. Digital Beamforming-Based Massive MIMO Transceiver for 5G MillimeterWave Communications. IEEE Trans. Microw. Theory Tech. 2018, 66, 3403-3418. [CrossRef]

6. Amani, N.; Glazunov, A.A.; Ivashina, M.V.; Maaskant, R. Per-Antenna Power Distribution of a Zero-Forcing Beamformed ULA in Pure LOS MU-MIMO. IEEE Commun. Lett. 2018, 22, 2515-2518. [CrossRef]

7. Bencivenni, C.; Glazunov, A.A.; Maaskant, R.; Ivashina, M.V. Aperiodic Array Synthesis for Multi-User MIMO Applications. arXiv 2018, arXiv:1808.08321.

8. Rusek, F.; Persson, D.; Lau, B.K.; Larsson, E.G.; Marzetta, T.L.; Edfors, O.; Tufvesson, F. Scaling Up MIMO: Opportunities and Challenges with Very Large Arrays. IEEE Signal Process. Mag. 2013, 30, 40-60. [CrossRef]

9. Amani, N.; Wymeersch, H.; Johannsen, U.; Smolders, A.B.; Ivashina, M.V.; Maaskant, R. Multi-Panel Sparse Base Station Design with Physical Antenna Effects in Massive MU-MIMO. IEEE Trans. Veh. Technol. 2020, 69, 6500-6510. [CrossRef]

10. SILIKA Project. Research Objectives. Available online: https://silika-project.eu/about-silika/research-objectives / (accessed on 11 January 2022).

11. Aslan, Y.; Puskely, J.; Janssen, J.H.J.; Geurts, M.; Roederer, A.; Yarovoy, A. Thermal-Aware Synthesis of 5G Base Station Antenna Arrays: An Overview and a Sparsity-Based Approach. IEEE Access 2018, 6, 58868-58882. [CrossRef]

12. Roev, A.; Maaskant, R.; Höök, A.; Ivashina, M. Efficient millimeter-wave high power generation with spatial power-combined feeding element. In Proceedings of the 12th European Conference on Antennas and Propagation (EuCAP 2018), London, UK, 9-13 April 2018; pp. 1-4. [CrossRef]

13. Vigano, M.C.; Toso, G.; Caille, G.; Mangenot, C.; Lager, I.E. Spatial density tapered sunflower antenna array. In Proceedings of the 2009 3rd European Conference on Antennas and Propagation, Berlin, Germany, 23-27 March 2009.

14. Bencivenni, C.; Ivashina, M.V.; Maaskant, R.; Wettergren, J. Synthesis of Maximally Sparse Arrays Using Compressive Sensing and Full-Wave Analysis for Global Earth Coverage Applications. IEEE Trans. Antennas Propag. 2016, 64, 4872-4877. [CrossRef]

15. Farsaei, A.; Amani, N.; Maaskant, R.; Gustavsson, U.; Alvarado, A.; Willems, F.M.J. Uniform Linear Arrays With Optimized Inter-Element Spacing for LOS Massive MIMO. IEEE Commun. Lett. 2021, 25, 613-616. [CrossRef]

16. Pinchera, D.; Migliore, M.D.; Schettino, F. Optimizing Antenna Arrays for Spatial Multiplexing: Towards 6G Systems. IEEE Access 2021, 9, 53276-53291. [CrossRef]

17. Pinchera, D.; Migliore, M.D.; Schettino, F.; Panariello, G. Antenna Arrays for Line-of-Sight Massive MIMO: Half Wavelength Is Not Enough. Electronics 2017, 6, 57. [CrossRef]

18. ITU. IMT Vision-Framework and Overall Objectives of the Future Development of IMT for 2020 and Beyond; Technical Report, ITU-R M.2083-0; International Telecommunication Union: Geneva, Switzerland, 2015.

19. White Paper: 5G Channel Model for Bands Up to $100 \mathrm{GHz}$ (Annex); Technical Report, Tech. Rep. Annex; Department of Computer Science, Michigan State University: East Lansing, MI, USA, 2016. 
20. Prinsloo, D.S.; Maaskant, R.; Ivashina, M.V.; Glazunov, A.A.; Meyer, P. Synergy in design of phased array antennas for modern radio astronomy and wireless communication systems. In Proceedings of the 201610 th European Conference on Antennas and Propagation (EuCAP), Davos, Switzerland, 10-15 April 2016; pp. 1-5. [CrossRef]

21. Moffet, A. Minimum-redundancy linear arrays. IEEE Trans. Antennas Propag. 1968, 16, 172-175. [CrossRef]

22. Bracewell, R.N. Radio astronomy techniques. In Handbuch Der Physik; Springer: Berlin/Heidelberg, Germany, 1962; Volume 54, pp. 42-129.

23. Silver, S. (Ed.) Microwave Antenna Theory and Design; Massachusetts Institute of Technology. Radiation Laboratory Series. No. 12; McGraw-Hill Book Company: New York, NY, USA, 1949.

24. Shapeways. Aluminum Material Information. Available online: https://www.shapeways.com/materials/aluminum (accessed on 11 January 2022).

25. Peel, C.B.; Hochwald, B.M.; Swindlehurst, A.L. A vector-perturbation technique for near-capacity multiantenna multiuser communication-part I: channel inversion and regularization. IEEE Trans. Commun. 2005, 53, 195-202. [CrossRef] 